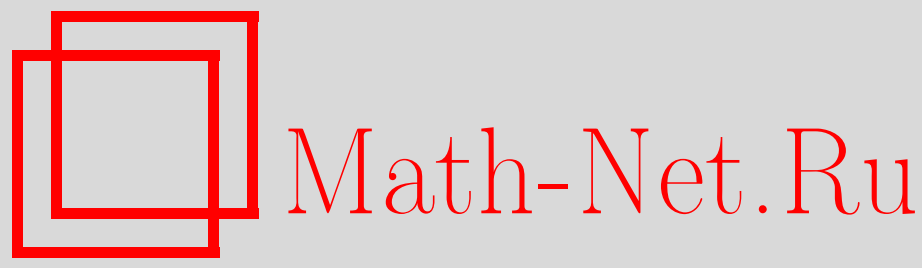

В. Н. Лихачев, Г. А. Виноградов, Функция Грина в задаче о динамике заряда на одномерной решетке с примесным центром, ТМФ, 2018, том 196, номер 1, 88-98

DOI: https://doi.org/10.4213/tmf9459

Использование Общероссийского математического портала Math-Net.Ru подразумевает, что вы прочитали и согласны с пользовательским соглашением http://www.mathnet.ru/rus/agreement

Параметры загрузки:

IP: 54.198 .64 .247

26 апреля 2023 г., 16:02:01

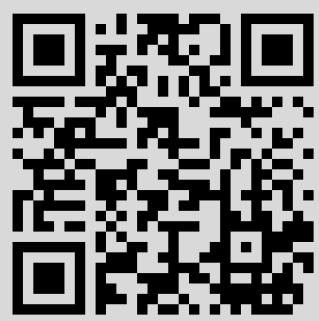




\title{
ФУНКЦИЯ ГРИНА В ЗАДАЧЕ О ДИНАМИКЕ ЗАРЯДА НА ОДНОМЕРНОЙ РЕШЕТКЕ С ПРИМЕСНЫМ ЦЕНТРОМ
}

\begin{abstract}
В приближении "сильной связи" (модель Хюккеля) рассмотрена эволюция волновой функции заряда на полубесконечной одномерной решетке с дополнительной энергией $U$ на единственном примесном узле. В случае непрерывного спектра (при $|U|<1$ ), когда отсутствует локализованное состояние, функция Грина построена с помощью разложения по собственным функциям непрерывного спектра. Выражение для временной функции Грина получено в виде степенного ряда по $U$. Неожиданно оказалось, что этот ряд сходится абсолютно даже в том случае, когда к непрерывному спектру добавляется локализованное состояние. Таким образом, можно сказать, что функция Грина, построенная по состояниям непрерывного спектра, имплицитно содержит и вклад локализованного состояния.
\end{abstract}

Ключевые слова: функция Грина, квантовая динамика.

DOI: https://doi.org/10.4213/tmf9459

\section{1. ВВЕДЕНИЕ}

Эффективным методом при изучении транспортных свойств неметаллических систем является так называемое приближение сильной связи (tight-binding approximation, tb-приближение) или, по-другому, приближение Хюккеля [1]-[3]. В этом приближении волновая функция представляется в виде линейной комбинации локализованных состояний. В частном случае молекулярных систем - в виде линейной комбинации атомных орбиталей. Тb-метод фактически является дискретной версией уравнения Шредингера. Используя этот подход, можно построить функцию Грина для исследуемой системы. Эта функция Грина, в свою очередь, позволяет вычислить транспортные свойства исследуемой системы.

Если система бесконечна и однородна, то спектр собственных значений непрерывен и функцию Грина можно построить путем разложения по собственным функциям tb-гамильтониана (см., например, работы [4]-[6]). Если система содержит дефекты, то подобно андерсоновской локализации возникают локализованные собствен-

* Институт биохимической физики им. Н. М. Эмануэля РАН, Москва, Россия. E-mail: gvin@deom.chph.ras.ru 


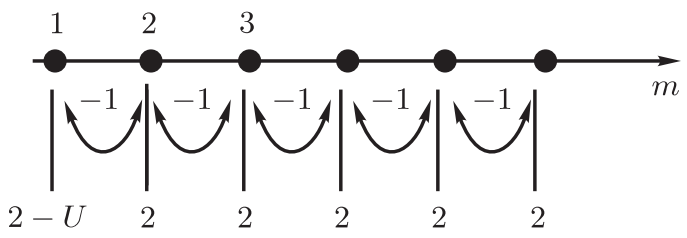

Рис. 1. Одномерная полубесконечная решетка с примесным центром на первом узле с дополнительной энергией $U$. Числа сверху представляют нумерацию атомов $(m \in[1, \infty])$. Значения снизу суть энергии на узлах. Полукруглые стрелки - интегралы перескока между соседними узлами и их энергии.

ные функции, и от непрерывного спектра отщепляются энергетические уровни, соответствующие этим локализованным функциям. В этом случае задача нахождения функции Грина становится существенно сложнее.

В настоящей работе рассмотрена задача о распространении квантового возбуждения (заряда) на одномерной решетке с единственным примесным центром в приближении сильно взаимодействующих электронов. Эта задача точно решена с помощью временной функции Грина. Получено решение, в котором выражение для функции Грина для бездефектной решетки удалось аналитически продолжить на решение для решетки с дефектом, когда от спектра отщепляется локализованный уровень. Ранее подобная задача с тем же tb-гамильтонианом была решена другим способом [7]-[9].

\section{2. ПОСТАНОВКА ЗАДАЧИ И РЕЗУЛЬТАТЫ}

Основная цель настоящей работы - получить решение для задачи об эволюции волновой функции на одномерной полубесконечной решетке с примесным центром при разных значениях степени дефектности примесного узла.

Рассмотрение проводится в рамках tb-приближения. В этой постановке задача имеет два параметра: интеграл перескока и энергия волновой функции на узле. Выберем значение интеграла перескока равным -1 , что одновременно означает выбор масштаба энергии. Энергию связи волновой функции с узлами решетки выберем равной +2 . Такое значение энергии означает, что дно валентной зоны соответствует нулевому значению энергии электрона. В решетке есть единственный примесный узел с дополнительной энергией $U$. Решетка полубесконечна и схематически показана на рис. 1.

Гамильтониан рассматриваемой задачи в матричном представлении имеет трехдиагональный вид

$$
H=\left(\begin{array}{ccccc}
\mathbf{2}-\mathbf{U} & -1 & 0 & 0 & \ldots \\
-1 & \mathbf{2} & -1 & 0 & \ldots \\
0 & -1 & \mathbf{2} & -1 & \ddots \\
0 & 0 & -1 & \mathbf{2} & \ddots \\
\vdots & \vdots & \ddots & \ddots & \ddots
\end{array}\right),
$$


где на главной диагонали стоят значения энергий (они выделены жирным шрифтом), а на двух побочных диагоналях - интегралы перескока. Решается безразмерное $(\hbar=1)$ нестационарное уравнение Шредингера для волновой функции $\Psi(m ; t)$ $i \partial_{t} \Psi=H \Psi$ при условии, что сначала электрон локализован на узле $m=m_{0}$, т. е. $\Psi(m ; t=0)=\delta_{m, m_{0}}$.

Если $|U|<1$, то спектр непрерывен, заключен в интервале энергий $[0,4]$, и отщепления уровней от спектра не происходит. В этом случае решение в виде функции Грина ищется стандартным образом путем разложения по собственным функциям гамильтониана (1). Собственные функции и энергии задачи (1) имеют вид (см. приложение A)

$$
\begin{gathered}
\phi_{k}(m)=\sqrt{\frac{2}{\pi}} \frac{1}{\sqrt{1-2 U \cos k+U^{2}}}\{\sin k m-U \sin [k(m-1)]\}, \\
E(k)=2[1-\cos k], \quad E(k) \in[0,4], \quad 0<k<\pi .
\end{gathered}
$$

Когда $|U|>1$, то появляется локализованное состояние $\phi_{\mathrm{loc}}(m)$ с энергией $E_{\mathrm{loc}}$ (см. приложение А)

$$
\phi_{\mathrm{loc}}(m)=\frac{\sqrt{U^{2}-1}}{U^{m}}, \quad E_{\mathrm{loc}}=2-U-\frac{1}{U},
$$

a энергия $E_{\mathrm{loc}}$ отщепляется от непрерывного спектра: $E_{\mathrm{loc}}<0$ или $E_{\mathrm{loc}}>4$ в зависимости от знака $U$.

В соответствии с известной формулой разложения решения по собственным функциям гамильтониана для случая $U<1$, когда отсутствует локализованное состояние, получаем представление функции Грина $G^{0}\left(m, m_{0} ; t\right)$

$$
G^{0}\left(m, m_{0} ; t\right)=\int_{0}^{\pi} d k \phi_{k}(m) \phi_{k}\left(m_{0}\right) e^{-i E(k) t} .
$$

Если же $|U|>1$, то нужно учесть связанное состояние (3), и тогда функция Грина принимает вид

$$
G^{*}\left(m, m_{0} ; t\right)=\frac{\left(U^{2}-1\right) e^{-i E_{\mathrm{loc}} t}}{U^{m+m_{0}}}+\int_{0}^{\pi} d k \phi_{k}(m) \phi_{k}\left(m_{0}\right) e^{-i E(k) t},
$$

где вклад локализованного состояния учитывается посредством дополнительного слагаемого. В общем случае функция Грина $G\left(m, m_{0} ; t\right)$ фактически представляет собой значение волновой функции на узле $m$ в момент времени $t$, если при $t=0$ волновая функция была целиком локализована на узле $m_{0}$, т. е. $\Psi(m ; t=0)=\delta_{m, m_{0}}$.

В случае отсутствия локализованного состояния $(|U|<1)$ можно получить представление функции $G^{0}\left(m, m_{0} ; t\right)$ в виде степенного ряда по $U$. Для этого в выражении (4) для собственных функций необходимо использовать разложение $e^{2 i t \cos x}=$ $\mathrm{J}_{0}(2 t)+2 \sum_{m=1}^{\infty} i^{m} \mathrm{~J}_{m}(2 t) \cos m x$ (см. работы $\left.{ }^{1)}[10]-[12]\right)$. Так как функция Грина (4)

\footnotetext{
1) Соответствующая формула в книге [10] приведена с опечаткой: вместо $\mathrm{J}_{0}$ стоит единица.
} 


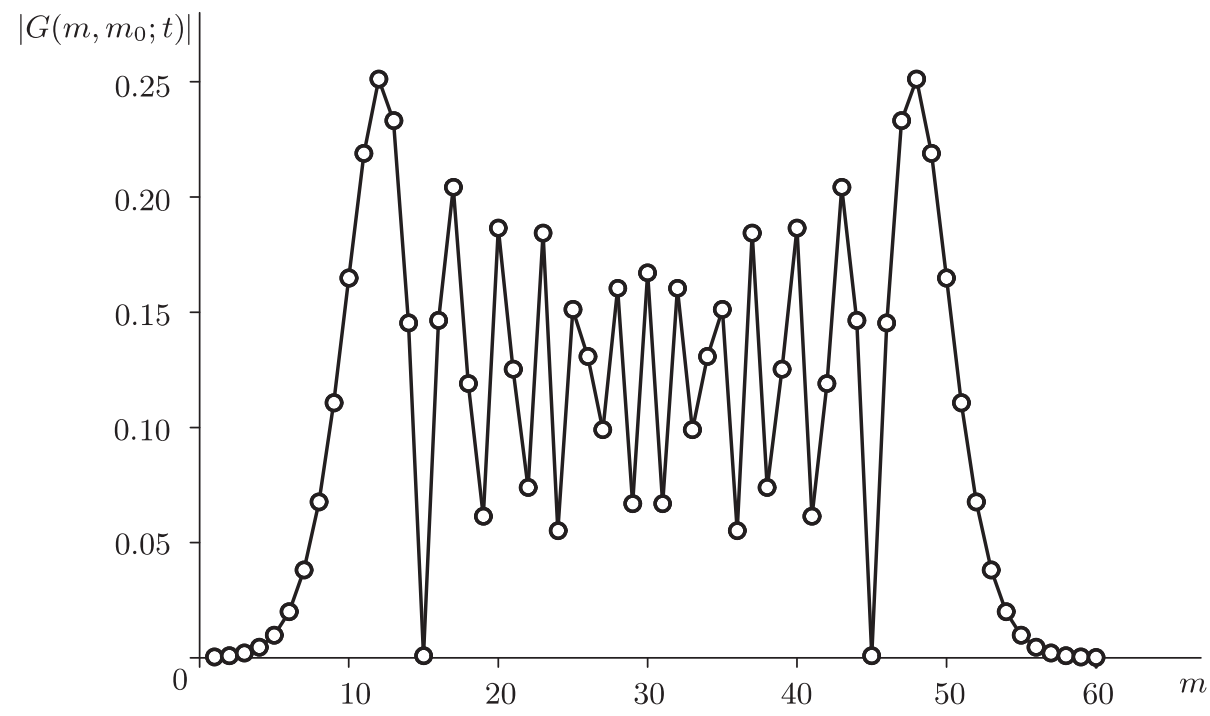

Рис. 2. Форма волнового пакета (модуль функции Грина для разных значений $m$ ) в момент времени $t=10$. При $t=0$ волновая функция локализована на узле $m_{0}=30$. Дополнительная энергия на примесном узле $U=2$. Пока импульс не достиг начала цепочки, его форма остается такой же, как и в случае бесконечной цепочки. Сплошная линия представляет ряд (6), круги результат вычисления интеграла (4). Разница между этими значениями имеет порядок $4 \cdot 10^{-13}$. Отличие от решения временного уравнения Шредингера имеет такую же точность. Скорость фронта импульса равна максимально возможной групповой скорости $v=2$, и за время $t=10$ фронт проходит расстояние $\approx 20$ узлов решетки в обе стороны от $m_{0}=30$.

симметрична относительно перестановки $m \leftrightarrow m_{0}$, то достаточно привести выражение только для случая $m \geqslant m_{0}$ :

$$
\begin{aligned}
& G^{0}\left(m, m_{0} ; t\right)=e^{-2 i t} i^{\left(m-m_{0}\right)} \times \\
& \quad \times\left\{\mathrm{J}_{m-m_{0}}(2 t)-(-1)^{m_{0}}\left[i U \mathrm{~J}_{m+m_{0}-1}(2 t)+\left(1-U^{2}\right) \sum_{j=0}^{\infty}(i U)^{j} \mathrm{~J}_{j+m+m_{0}}(2 t)\right]\right\} .
\end{aligned}
$$

На рис. 2 показана функция Грина в момент времени $t=10$. На следующем рис. 3 для сравнения показана функция Грина в момент времени $t=40$.

Удивительным образом оказывается, что ряд (6), полученный для случая $|U|<1$, является точным также и для случая $|U|>1$, т. е. совпадает с выражением (5).

Заметим, что непосредственное суммирование ряда (6) для больших $t$ приводит к расходимости, связанной с конечной точностью вычислений. Здесь ситуация такая же, как с разложением в ряд функции $e^{i x}$ : при $x \gg 1$ ряд расходится. В приложении Б приведено два способа вычисления ряда (6), годящихся и для больших времен. 


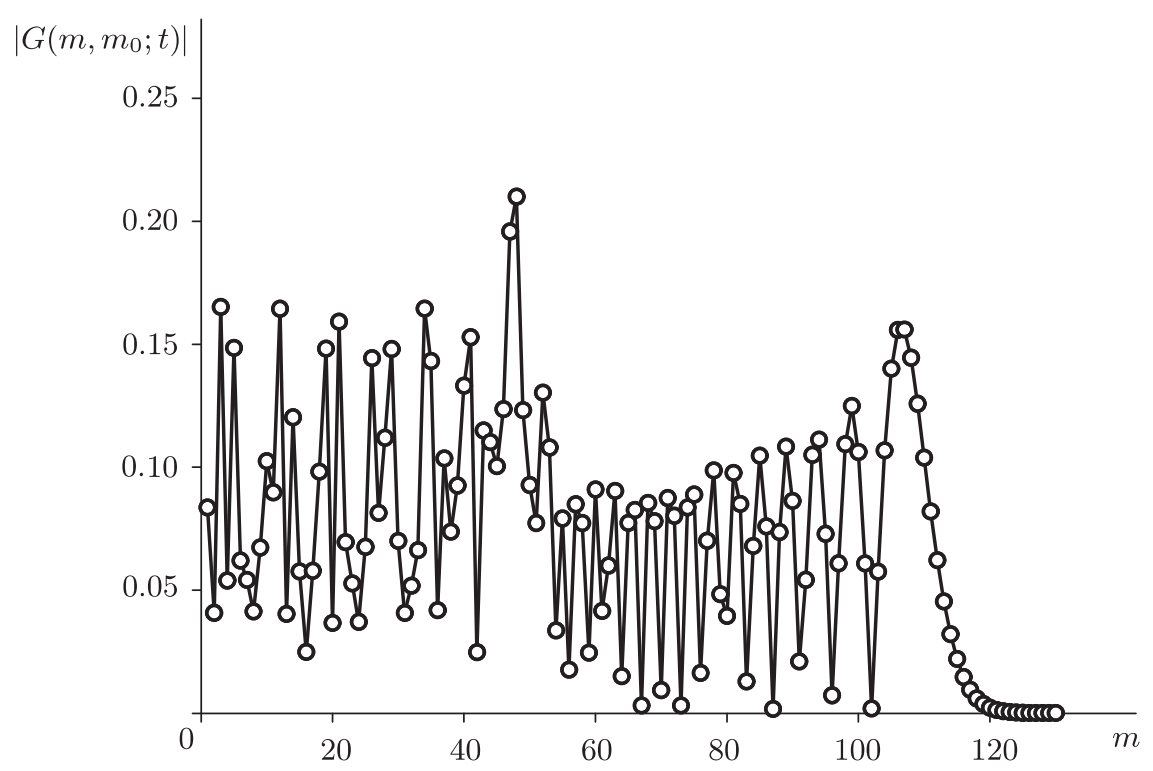

Рис. 3. Форма волнового пакета в момент времени $t=40$. При $t=0$ волновая функция локализована на узле $m_{0}=30$. Параметр $U=0.5$. Сплошная линия отображает ряд (6); круги - результат вычисления интеграла (4). Разница между этими значениями имеет порядок $10^{-11}$. Отличие от решения временного уравнения Шредингера имеет такую же точность. За время $t=40$ фронт импульса, распространяющийся направо и движущийся со скоростью $v=2$, достигает узла $m=110$. Фронт импульса, первоначально движущийся налево, за это же время отражается от левой границы решетки и, двигаясь направо, достигает узла $m=50$.

Действительно, вычисление интеграла в (5) (случай $|U|>1$ ) приводит к степенному ряду, но уже по обратным степеням $U$. Обозначив $V \equiv 1 / U$, получаем выражение

$$
\begin{aligned}
G^{*}\left(m, m_{0} ; t\right)= & \frac{U^{2}-1}{U^{m+m_{0}}} e^{-i E_{\mathrm{loc}} t}+ \\
& +e^{-2 i t}\left[\left(i^{m-m_{0}}\right) \mathrm{J}_{m-m_{0}}(2 t)+\left(i^{m+m_{0}-1}\right) V \mathrm{~J}_{m+m_{0}-1}(2 t)\right]+ \\
& +e^{-2 i t}\left(V^{2}-1\right) V^{m+m_{0}-2}\left[\sum_{j=1}^{\infty}(i V)^{j} \mathrm{~J}_{j}(2 t)+\sum_{j=0}^{m+m_{0}-2}(i U)^{j} \mathrm{~J}_{j}(2 t)\right] .
\end{aligned}
$$

Как и ранее, в случае $|U|>1$, непосредственное суммирование ряда в (7) приводит к расходимостям из-за конечной точности вычислений (см. приложение Б).

Полученный ряд (7) является просто другой формой записи ряда (6). Для доказательства совпадения выражений (6) и (7) для функций Грина вклад от локализованного состояния в выражении (7) нужно преобразовать с помощью производящей функции для функций Бесселя [10]: $e^{-i E_{\mathrm{loc}} t}=e^{-2 i t} \sum_{j=-\infty}^{\infty}(i U)^{j} \mathrm{~J}_{j}(2 t)$. 


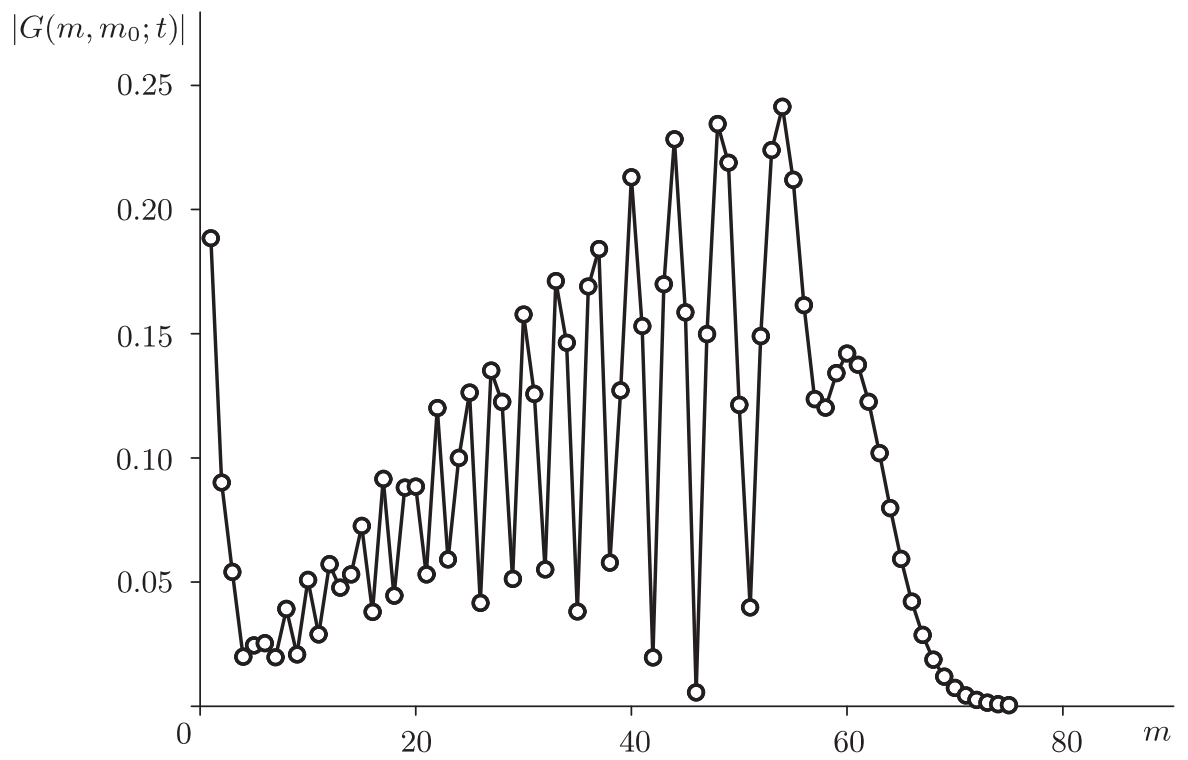

Рис. 4. Форма волнового пакета в момент времени $t=30$. При $t=0$ волновая функция локализована на узле $m_{0}=3$. Параметр $U=2$. Сплошная линия отображает ряд (7); круги суть результат вычисления интеграла (5). Разница между этими значениями имеет порядок $10^{-10}$. Отличие от решения временного уравнения Шредингера имеет такую же точность. Кроме уходящего направо имульса, есть еще часть волновой функции, оставшейся в локализованном состоянии. Форма импульса представляет собой суперпозицию импульса, исходно уходящего направо, и импульса, отраженного от начала решетки.

Так как полученный результат представляется довольно неожиданным, было численно проверено, что ряд (6) действительно правильно воспроизводит исходные выражения (4) и (5). Проверка осуществлялась как путем вычисления интегралов (4), (5), так и путем решения временного уравнения Шредингера, вытекающего из (1). На рис. 4 показана функция Грина в момент $t=30$.

На рис. 5 показана зависимость от времени функции Грина на фиксированном узле $m=5$.

\section{3. ЗАКЛЮЧЕНИЕ}

В заключение мы считаем возможным предположить, что полученный в рассмотренной задаче результат может носить более общий характер. А именно, пусть рассматривается "стандартная" квантовомеханическая задача с потенциалом, зависящим от параметра. И пусть у этого параметра есть пороговое значение, при котором появляется связанное состояние. Можно предположить, что функция Грина, полученная в допороговой области разложением только по собственным функциям 


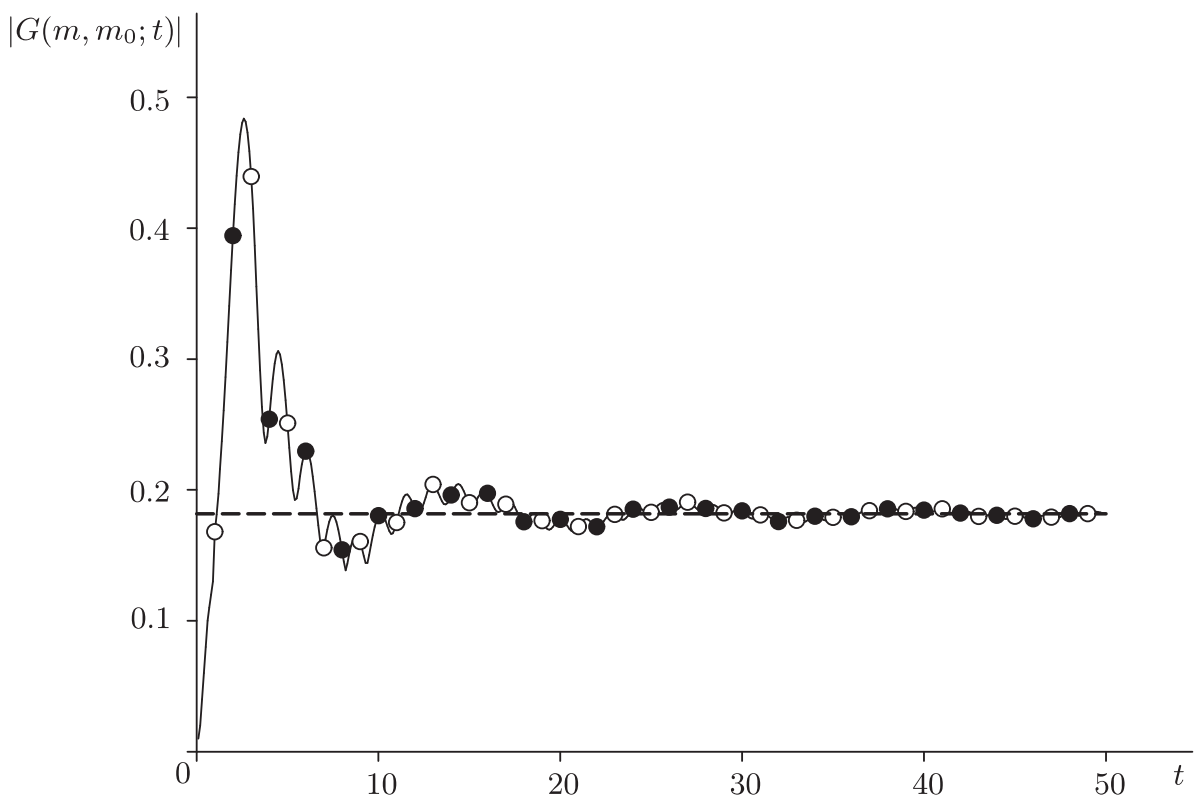

Рис. 5. Зависимость от времени функции Грина на узле $m=5$. Параметры $U=2, m_{0}=1$. При $t \rightarrow \infty$ модуль функции выходит на постоянное значение $\phi_{\mathrm{loc}}(1) \phi_{\mathrm{loc}}(5)=0.18175$. Сплошная линия отображает формулу (7), светлые круги - результат вычисления интеграла в (5), черные круги - результат решения уравнения Шредингера. Разница между всеми этими значениями имеет порядок $10^{-12}$.

непрерывного спектра, дает правильный ответ и в послепороговой области, где учитывается локализованное состояние. Другими словами, степенной ряд по параметру, представляющий собой функцию Грина, сходится всегда, т. е. не имеет каких-либо особенностей при критическом значении параметра, отвечающем появлению локализованного состояния.

ПРИЛОЖЕНИЕ А Нахождение собственных функций гамильтониана (1)

Уравнение Шредингера на собственные значения гамильтониана (1) имеет вид

$$
2 \phi(m)-\phi(m-1)-\phi(m+1)=E \phi(m), \quad m=2,3, \ldots .
$$

Решение можно искать в виде $\phi(m) \sim a \sin k m+b \cos k m$. Подставляя это выражение в (А.1), получаем выражение для энергии

$$
E(k)=2(1-\cos k) .
$$

Для примесного узла $m=1$ уравнение Шредингера имеет вид

$$
(2-U) \phi(1)-\phi(2)=E \phi(2) \text {. }
$$


Можно убедиться, что уравнению (А.3) удовлетворяет следующая собственная функция:

$$
\phi_{k}(m)=A(k)\{\sin k m-U \sin [k(m-1)]\}, \quad 0<k<\pi .
$$

Нормировочный множитель $A(k)$ находится из условия ортогональности

$$
\left\langle\phi_{k_{1}} \mid \phi_{k_{2}}\right\rangle=\delta\left(k_{1}-k_{2}\right) \text {. }
$$

Таким образом, нужно, чтобы было выполнено равенство

$$
\begin{aligned}
A\left(k_{1}\right) A\left(k_{2}\right) \sum_{m=1}^{\infty}\{ & \left.\sin k_{1} m-U \sin \left[k_{1}(m-1)\right]\right\} \times \\
& \times \sum_{m=1}^{\infty}\left\{\sin k_{2} m-U \sin \left[k_{2}(m-1)\right]\right\}=\delta\left(k_{1}-k_{2}\right) .
\end{aligned}
$$

После перемножения отдельных членов в формуле (А.6) возникает несколько комбинированных сумм. Вычислим их по отдельности. Первая сумма есть

$$
S_{1} \equiv \sum_{m=1}^{\infty} \sin k_{1} m \sin k_{2} m=\frac{1}{2} \sum_{m=1}^{\infty}\left\{\cos \left[\left(k_{1}-k_{2}\right) m\right]-\cos \left[\left(k_{1}+k_{2}\right) m\right]\right\} .
$$

Воспользовавшись известным соотношением $\sum_{m=1}^{\infty} \cos m x=\pi \delta(x)-1 / 2$, получаем равенство $S_{1}=\pi\left[\delta\left(k_{1}-k_{2}\right)+\delta\left(k_{1}+k_{2}\right)\right] / 2$. Из-за того, что волновое число $k>0$, в сумме $S_{1}$ остается только первое слагаемое:

$$
S_{1}=\frac{\pi}{2} \delta\left(k_{1}-k_{2}\right)
$$

Для второй суммы аналогично получаем, что

$$
S_{2} \equiv \sum_{m=1}^{\infty} U^{2}\left\{\sin \left[k_{1}(m-1)\right] \sin \left[k_{2}(m-1)\right]\right\}=\frac{\pi U^{2}}{2} \delta\left(k_{1}-k_{2}\right)
$$

Третью сумму $S_{3} \equiv-U \sum_{m=1}^{\infty} \sin k_{1} m \sin \left[k_{2}(m-1)\right]$ представим в виде

$$
\begin{aligned}
S_{3}= & \frac{U}{2} \sum_{m=1}^{\infty} \cos k_{2}\left\{\cos \left[\left(k_{1}+k_{2}\right) m\right]-\cos \left[\left(k_{1}-k_{2}\right) m\right]\right\}+ \\
& +\frac{U}{2} \sum_{m=1}^{\infty} \sin k_{2}\left\{\sin \left[\left(k_{1}+k_{2}\right) m\right]+\sin \left[\left(k_{1}-k_{2}\right)\right\} .\right.
\end{aligned}
$$

Первая сумма в (А.10) равна $-\pi U \cos k_{2} \delta\left(k_{1}-k_{2}\right) / 2$, слагаемое с $\delta\left(k_{1}+k_{2}\right)$, как и ранее, опускаем. Вторая сумма вычисляется с помощью соотношения $\sum_{m=1}^{\infty} \sin m x=$ $[\operatorname{ctg}(x / 2)] / 2$. В результате получаем, что

$$
S_{3}=-\frac{\pi U}{2} \cos k_{1} \delta\left(k_{1}-k_{2}\right)+\frac{U}{4} \sin k_{2}\left[\operatorname{ctg} \frac{k_{1}+k_{2}}{2}+\operatorname{ctg} \frac{k_{1}-k_{2}}{2}\right] .
$$

Выражение для суммы $S_{4} \equiv-U \sum_{m=1}^{\infty} \sin k_{2} m \sin \left[k_{1}(m-1)\right]$ получается из формулы (А.11) перестановкой $k_{1} \leftrightarrow k_{2}$ :

$$
S_{4}=-\frac{\pi U}{2} \cos k_{2} \delta\left(k_{2}-k_{1}\right)+\frac{U}{4} \sin k_{1}\left[\operatorname{ctg} \frac{k_{2}+k_{1}}{2}+\operatorname{ctg} \frac{k_{2}-k_{1}}{2}\right] .
$$


При сложении $S_{3}+S_{4}$ вторые слагаемые сокращаются, и мы приходим к равенству

$$
S_{3}+S_{4}=-\frac{\pi U}{2}\left(\cos k_{1}+\cos k_{2}\right) \delta\left(k_{1}-k_{2}\right) .
$$

Складывая $S_{1}+S_{2}+S_{3}+S_{4}$, окончательно имеем

$$
S_{1}+S_{2}+S_{3}+S_{4}=-\frac{\pi}{2}\left[1+U^{2}-U\left(\cos k_{1}+\cos k_{2}\right)\right] \delta\left(k_{1}-k_{2}\right) .
$$

Подставляя это выражение в (А.6), для нормировочного множителя $A(k)$ получаем соотношение

$$
A^{2}(k)=\frac{\pi}{2}\left[1+U^{2}-2 U \cos k\right] .
$$

Отсюда выражение для нормированной собственной функции имеет окончательный вид

$$
\phi_{k}(m)=\sqrt{\frac{2}{\pi}} \frac{1}{\sqrt{1-2 U \cos k+U^{2}}}\{\sin k m-U \sin [k(m-1)]\} .
$$

Отметим, что функция (А.16) (в случае $|U|<1$ ) удовлетворяет условию полноты

$$
\int_{0}^{\pi} \phi_{k}(m) \phi_{k}(n) d k=\delta_{m, n}
$$

Заметим также, что уравнению на собственные значения (А.1) очевидным образом удовлетворяет экспоненциально убывающая функция $\phi_{m} \sim q^{m}$, где $|q|<1$. Энергия этого состояния $E=2-q-1 / q$. При подстановке этого решения в уравнение (А.3) для первого узла, получаем, что $q=1 / U$. Нормируя это состояние на единицу, получаем следующее выражение для локализованного состояния:

$$
\phi_{\mathrm{loc}}(m)=\frac{\sqrt{U^{2}-1}}{U^{m}}, \quad E_{\mathrm{loc}}=2-U-\frac{1}{U} .
$$

Задача по нахождению собственных значений и векторов в более сложной геометрии, но для случая отсутствия дефектов, была ранее уже рассмотрена [11].

\section{ПРИЛОЖЕНИЕ Б}

\section{Некоторые подробности вычисления ряда (6)}

Сделаем дополнительное замечание относительно сходимости ряда (6) и возможности его вычислить. Для исследования сходимости рассмотрим вспомогательный ряд, который входит в выражение (6) для функции Грина:

$$
F(U, t)=\sum_{j=0}^{\infty}(i U)^{j} \mathrm{~J}_{j}(2 t) .
$$

Этот ряд сходится абсолютно при любых значениях $U$ и $t$. Действительно, при больших значениях индекса $j$ у функции Бесселя справедлива следующая аппроксимация (если опустить предэкспоненциальный множитель): $\mathrm{J}_{j}(t) \simeq e^{j(\operatorname{th}(\alpha)-\alpha)}$, где $\operatorname{ch} \alpha=j / t, j \gg 1$ и $j>t$.

Если $j \gg t$, то

$$
\mathrm{J}_{j} \simeq \exp \left\{j\left[1-\ln \frac{2 j}{t}+O\left(\frac{t}{j}\right)\right]\right\}
$$




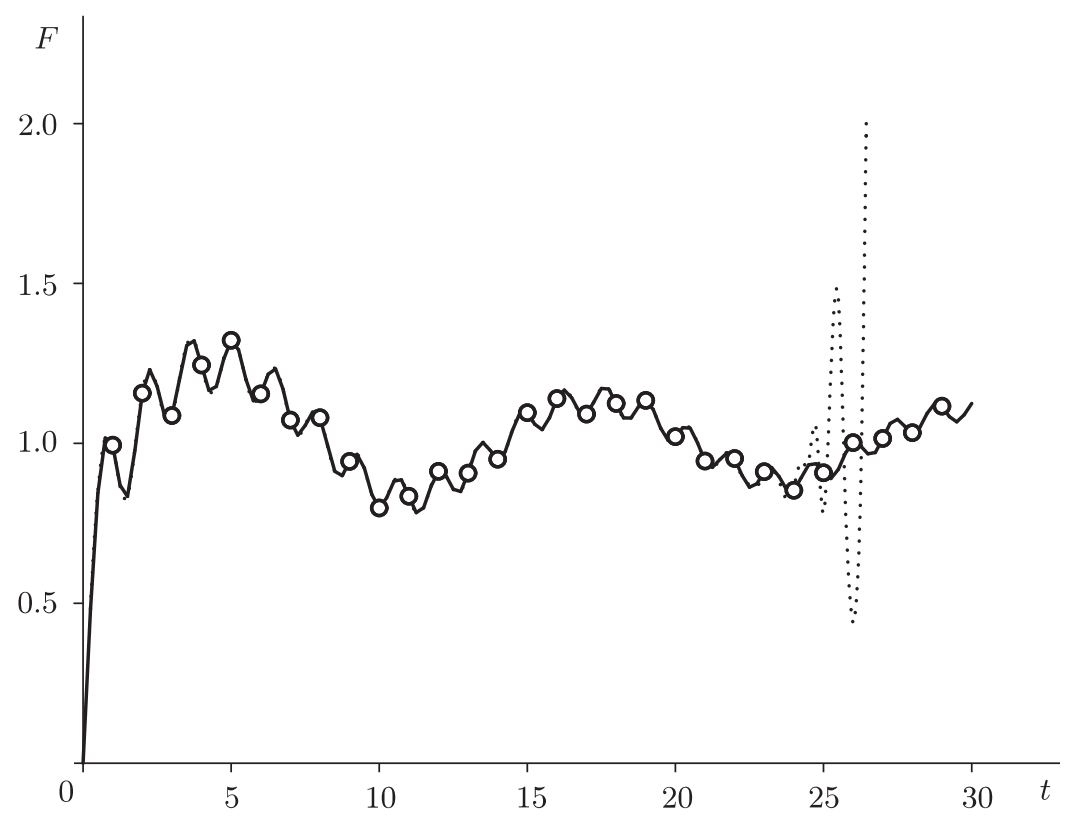

Рис. 6. Зависимость функции $F$ от времени. Параметр $U=2$. Сплошная линия отображает результат вычисления по формуле (Б.2); кругами изображен результат вычисления интеграла (Б.4). Максимальное расхождение имеет порядок $10^{-11}$. Точками показан результат прямого суммирования в формуле (Б.1), которое приводит к расходимости для больших значений $t$.

Таким образом, функция Бесселя $\mathrm{J}_{j}(t)$ убывает по индексу $j$ быстрее, чем экспонента. Следовательно, ряд (Б.1) сходится абсолютно.

Следует сделать еще одно замечание относительно эффективности численного подсчета ряда (Б.1). Непосредственное суммирование в случае $|U|>1$ может дать неправильный результат из-за конечной точности вычислений. Действительно, рассматриваемый ряд (Б.1) является знакопеременным (по вещественной и мнимой частям). При этом отдельные члены ряда (в случае $|U|>1$ и $t \gg 1$ ) могут достигать больших (по модулю) значений.

Для этого ряда максимальное по $j$ значение $F_{j} \equiv U^{j} \mathrm{~J}_{j}(2 t)$ равно

$$
F_{j}=\frac{1}{\sqrt{2 \pi t(U-1 / U)}} e^{t(U-1 / U)}
$$

(оно достигается при $j \sim t(U+1 / U)$ ). И если это значение имеет порядок обратной точности вычислений, то ответ становится неверным. Стандартная точность вычислений на языке FORTRAN имеет порядок $10^{-15}$. Если выбрать $U=2$, то при $t \gtrsim 25$ ответ становится неточным, что видно на рис. 6 . Поэтому в случае $|U|>1$ для вычисления суммы (Б.1) следует использовать хорошо сходящийся ряд по $1 / U$. 
Для этого нужно взять производящую функцию для функций Бесселя

$$
\exp \left[i t\left(U+\frac{1}{U}\right)\right]=F(U, t)+F\left(U^{-1}, t\right)+\mathrm{J}_{0}(2 t) .
$$

Собственно говоря, использование именно этого соотношения позволяет убедиться в тождественности формул (6) и (7). Заметим, что в работе [10] (см. также книгу [12]) для этой формулы ошибочно указано ограничение $2 t<|U|$.

Для вычисления ряда (Б.1) для любых значений $U$ можно использовать дифференциальное уравнение. Продифференцировав (Б.1) по времени и воспользовавшись соотношением $2 d J_{j} / d t=\mathrm{J}_{j-1}-\mathrm{J}_{j+1}$, получаем равенство

$$
\frac{d F}{d t}=i \omega F+i U \mathrm{~J}_{0}(2 t)+\mathrm{J}_{1}(2 t), \quad \omega \equiv U+\frac{1}{U}, \quad F(0)=1 .
$$

Решение этого уравнения можно записать в следующем виде:

$$
F=\frac{1}{2} e^{i \omega t}-\mathrm{J}_{0}(2 t)+\frac{i}{2}\left(U+\frac{1}{U}\right) e^{i \omega t} \int_{0}^{t} e^{-i \omega \tau} \mathrm{J}_{0}(2 \tau) d \tau .
$$

Функция $F$, полученная по приведенным выше формулам, показана на рис. 6 .

\section{Список литературы}

[1] G. Cuniberti, G. Fagas, K. Richter, "Fingerprints of mesoscopic leads in the conductance of a molecular wire", Chem. Phys., 281:2-3 (2002), 465-476.

[2] T. N. Todorov, "Tight-binding simulation of current-carrying nanostructures", J. Phys.: Condens. Matter, 14:11 (2002), 3049-3084.

[3] R. Movassagh, G. Strang, Y. Tsuji, R. Hoffman, "The Green's function for the Hückel (tight binding) model", J. Math. Phys., 58:3 (2017), 033505, 20 pp.

[4] K. D. Sattler (ed.), Fundamentals of Picoscience, CRC Press, Boca Raton, FL, 2014.

[5] D. A. Ryndyk, R. Gutiérrez, B. Song, G. Cuniberti, "Green function techniques in the treatment of quantum transport at the molecular scale", Energy Transfer Dynamics in Biomaterial Systems, Springer Series in Chemical Physics, 93, eds. I. Burghardt, V. May, D. A. Micha, E. R. Bittner, Springer, Berlin, Heidelberg, 2009, 213-335.

[6] Ю.А. Кругляк, Наноэлектроника “снизу - вверх", Изд-во Стрельбицкого, Киев, 2016.

[7] В.Н. Лихачев, Т. Ю. Астахова, Г.А. Виноградов, “'Электронный пинг-понг” на одномерной решетке. Движение волнового пакета до первого отражения", ТМФ, 175:2 (2013), 279-299.

[8] В.Н.Лихачев, Т. Ю. Астахова, Г. А. Виноградов, “Электронный пинг-понг” на одномерной решетке. Многократные отражения волнового пакета и захват волновой функции акцептором", ТМФ, 176:2 (2013), 306-321.

[9] V.N. Likhachev, O.I. Shevaleevskii, G. A. Vinogradov, "Quantum dynamics of charge transfer on the one-dimensional lattice: wave packet spreading and recurrence", Chinese Phys. B, 25:1 (2016), 018708.

[10] И.С. Градштейн, И.М. Рыжик, Таблицы интегралов, сумм, рядов и произведений, Физматгиз, М., 1962.

[11] N. J. Balmforth, P. J. Morrison, "Normal modes and continuous spectra", Ann. N. Y. Acad. Sci., 773, no. 1, Waves in Astrophysics, 1995, 80-94.

[12] Р. О. Кузьмин, Бесселевы функции, ОНТИ, М.-Л., 1935.

Поступила в редакцию 5.09.2017, после доработки 20.10.2017 